\title{
Análise espacial da tuberculose infantil no Estado do Espírito Santo, 2000 a 2007
}

\author{
Spatial analysis on childhood tuberculosis in the State of Espirito Santo, Brazil, 2000 to 2007 \\ Carolina Maia Martins Sales ${ }^{1,2}$, Túlio Alberto Martins de Figueiredo ${ }^{1,2}$, Eliana Zandonade ${ }^{1,3}$
e Ethel Leonor Noia Maciel ${ }^{1,2,4}$
}

\begin{abstract}
RESUMO
Introdução: A tuberculose infantil é responsável por 15\% das notificações dos casos. O foco do Programa Nacional de Controle da Tuberculose é a identificação da tuberculose em adultos, deixando os menores de 15 anos à margem dos estudos, diagnóstico e tratamento. A análise espacial quantifica a exposição à doença e expõe as principais causas relacionadas ao espaço geográfico. O objetivo deste estudo é analisar a distribuição espacial da tuberculose infantil no Espírito Santo, de 2000 a 2007, segundo município de notificação. Métodos: Realizou-se um estudo ecológico dos 515 casos de tuberculose infantil, entre 2000 e 2007. Utilizou-se o Método Bayesiano Empírico Local para estimação do risco. O Índice de Moram Local foi calculado para avaliação da autocorrelação entre as incidências de bairros limítrofes. Resultados: Altas taxas de incidência foram encontradas na Região Metropolitana de Vitória e região nordeste, e baixas taxas na região serrana; dados semelhantes foram observados no estudo da endemia de tuberculose em adultos no Espírito Santo, o que pode possivelmente estar relacionada aos contatos intradomiciliares. Conclusões: Este estudo identifica possíveis áreas de transmissão recente da doença. É importante ressaltar que o conhecimento dos locais prioritários para o controle, pode auxiliar a gestão publica na diminuição das iniquidades em saúde e permitir uma otimização dos recursos e das equipes no controle da tuberculose infantil.

Palavras-chaves: Tuberculose infantil. Análise espacial. Transmissão.
\end{abstract}

\begin{abstract}
Introduction: Childhood tuberculosis is responsible for $15 \%$ of case notifications. The focus of Tuberculosis Control National Program is on identifying tuberculosis in adults, while leaving children under 15 years of age on the margins of studies, diagnoses and treatment. Spatial analysis quantifies the exposition to the illness and displays the main causes relating to geographical space. The objective of this study was to analyze the spatial distribution of childhood tuberculosis in Espírito Santo, between 2000 and 2007, according to the municipality of notification. Methods: An ecological study was conducted on 515 cases of childhood tuberculosis that occurred between 2000 and 2007. The Local Empirical Bayesian Method was used to measure the risk. The Moran Local Index was calculated in order to evaluate autocorrelations between threshold districts. Results: High incidence rates were found in the Metropolitan Region of Vitória and the northeastern region, and lower rates were found in the southeastern region. Similar data were observed in a study on endemic tuberculosis among adults in Espírito Santo. This is possibly related to contacts within the home. Conclusions: This study identified possible areas of recent transmission of the disease. It is important to emphasize that knowledge of the high priority areas for tuberculosis control may help public administrators to diminish healthcare iniquities and enable improvement of resources and teams for controlling childhood tuberculosis.
\end{abstract}

Key-words: Childhood tuberculosis. Spatial analysis. Transmission.

1. Programa de Pós Graduação em Saúde Coletiva, Universidade Federal do Espírito Santo, Vitória, ES 2. Departamento de Enfermagem, Universidade Federal do Espírito Santo, Vitória, ES. 3. Departamento de Estatística, Universidade Federal do Espírito Santo, Vitória, ES. 4. Núcleo de Doenças Infecciosas, Universidade Federal do Espírito Santo, Vitória, ES.

Endereço para correspondência: Dra. Ethel Leonor Noia Maciel. NDI/UFES. Av Marechal Campos 1468, 29040-090 Vitória, ES.

e-mail: emaciel@ndi.ufes.br

Tel: 5527 3335-7210

Recebido para publicação em 08/03/2010

Aceito em 04/06/2010

\section{INTRODUÇÃO}

A tuberculose atinge 9 milhões de pessoas e mata 2 milhões, anualmente, no mundo ${ }^{1}$. Em países em desenvolvimento, ocorrem, aproximadamente, 1.300.000 casos e 450.000 mortes por tuberculose em crianças menores de 15 anos $^{3}$, anualmente 2 . Estima-se que do total de casos notificados no Brasil, $15 \%$ representam crianças nessa faixa etária ${ }^{3}$. No Espírito Santo, nos anos de 1990 a 2001, 1.284 casos de tuberculose infantil foram notificados ${ }^{4}$.

Os sinais e sintomas da tuberculose na infância são inespecíficos, o que dificulta a suspeição clínica e retarda o diagnóstico da doença. Seu espectro clínico é extremamente variado e vai desde formas assintomáticas até formas graves disseminadas da doença com emagrecimento significativo e evolução para o óbito 5 .

Devido às dificuldades diagnósticas apontadas acima, vários sistemas de pontuação (escores) foram desenvolvidos ${ }^{5-8}$. Entretanto, estes instrumentos apresentam uma grande variação na sua sensibilidade e especificidade e nos seus valores preditivos. Recentemente, o Ministério da Saúde do Brasil desenvolveu um Sistema de Escore Para o Diagnóstico de Tuberculose Pulmonar em Crianças e Adolescentes e recomendou sua utilização nos serviços onde esta faixa é atendida9.

A adoção deste sistema de pontuação pelos serviços de saúde visa melhorar as estatísticas de notificação da doença. No Estado do Espírito Santo, onde a doença é endêmica, o problema da subnotificação pode ficar mais evidente em estudos com dados geoprocessados, onde áreas pequenas aparecem com taxas de incidência igual a zero.

A análise espacial utiliza o geoprocessamento e os sistemas de informações geográficas, e tem como finalidade, através do mapeamento da doença em determinado espaço, obter taxas ajustadas.

Diante do exposto, este estudo tem por objetivo analisar a distribuição espacial da tuberculose infantil no Estado do Espírito Santo, no período de 2000 a 2007 e obter taxas ajustadas da incidência da doença. 


\section{MÉTODOS}

Trata-se de um estudo ecológico de dados secundários. Foram utilizados os casos de tuberculose infantil do Estado do Espírito Santo, referentes ao período de janeiro de 2000 a dezembro de 2007, notificados no banco de dados do Sistema de Informações sobre Agravos de Notificação da Secretaria de Vigilância Sanitária do Ministério da Saúde (SINAN/SVS/MS). Para o georeferenciamento das informações e análise espacial, foi utilizada a malha digital do Estado do Espírito Santo, no ano de 2000, fornecida pelo GEOBASES ${ }^{10}$. Os arquivos eram compatíveis com o software TerraView 3.2.0.

A endemia de tuberculose foi caracterizada pelas taxas de incidência de novos casos para a doença e foram calculadas seguindo a metodologia utilizada pela SVS/MS ${ }^{11}$ : taxas de incidência de tuberculose infantil foram calculadas dividindo-se o número de casos novos notificados em cada município pela população da área, menores de 15 anos para os anos estudados por 100 mil habitantes.

Para uma maior estabilidade das taxas, utilizou-se o somatório do número de casos novos dos anos de estudo (2000 a 2007), e das populações segundo município de notificação do caso novo notificado, neste mesmo período.

Para a correção de taxas epidemiológicas, utilizou-se a estatística espacial. O primeiro método utilizado foi a média móvel espacial. O segundo método foi a suavização da flutuação associada a áreas pequenas - estimador bayesiano empírico local ${ }^{12}$.

Seguindo a proposição, o estimador bayesiano empírico é uma taxa ajustada na forma:

$$
b i=w_{i} t_{i}+\left(l-w_{i}\right) m
$$

onde té a taxa de risco ou taxa de incidência de tuberculose na área, $m$ é taxa global para o Estado do Espírito Santo e $w_{i}$ é o peso entre 0 e 1 que depende do tamanho da população da área $i^{12}$. Quanto maior a população da área $i$, mais próximo de 1 é o peso $w_{i}$, o que implica que nas áreas cuja população é maior, a estimativa bayesiana empírica é muito próxima da taxa $t_{i}$ e nas áreas com população muito pequena, o valor de $b_{i}$ será intermediário entre $t_{i} \mathrm{e} m^{12}$.

No estudo de Análise dos Padrões Espaciais de Ocorrência, utilizou-se a autocorrelação espacial entre as taxas a fim de investigar se a distribuição espacial da endemia de tuberculose infantil se dá de forma aleatória ou segue algum padrão de ocorrência no espaço. Para tal, foi calculado o Índice Global de Moran I e o Índice de Moran Local (LISA), entendendo que os valores de significância inferiores a 0,05 delimitam regiões onde existem estruturas espaciais locais de surgimento da TB infantil ${ }^{13}$.

O Índice de Moran é dado por:

$$
I=\frac{{ }^{n} \sum_{i \neq j} W_{i j}\left(Y_{i}-\bar{Y}\right)\left(Y_{j}-\bar{Y}\right)}{S_{0} \sum_{i=j}^{n}\left(Y_{i}-\bar{Y}\right)^{2}}
$$

$$
\text { onde: } \quad S_{0}=\sum i \neq j W_{i j}
$$

Onde Y1, Y2, ::: Yn são variáveis aleatórias medidas em $n$ áreas e Wij são medidas da matriz de pesos W. Quando o valor de I é próximo de zero podemos concluir que há pouca correlação espacial, ou seja, indica fraca ou nula dependência espacial. Quando há valores de I positivo há indício de presença de dependência espacial e valores negativo para I não são comuns ${ }^{13}$.
O indicador LISA é expresso pela seguinte fórmula ${ }^{13}$ :

$$
I=\sum_{j=1}^{n} W_{i j} Z_{j} Z_{i} / \sum_{j=1}^{n} Z_{j}^{2}
$$

A estatística calculada para o índice LISA é computada de forma similar ao índice global de $I$ de Moran onde $\mathrm{Z}$ é a variável Y padronizada pela média. Portanto, deve ser averiguado se os respectivos valores de significância, em relação à hipótese nula (independência espacial), são menores que $0,05^{13}$.

Para detectar a significância, utiliza-se a ferramenta de visualização pelo mapa de significância LISA, onde são mapeadas e destacadas as associações significantes $(\mathrm{p} \leq 0,05)^{13}$.

\section{Ética}

Para a realização deste estudo, o projeto foi previamente submetido à apreciação e aprovação pelo Comitê de Ética em Pesquisa do Centro de Ciência da Saúde da Universidade Federal do Espírito Santo.

\section{RESULTADOS}

Dos 515 sujeitos da pesquisa (Tabela 1), observamos que a faixa etária predominante foi entre 1 e 5 anos. Em relação ao sexo das crianças e adolescentes notificados, 49,9\% (257/515) eram masculino e 50,1\% (258/515) femininos. Residem em municípios prioritários para o Controle da Tuberculose (Vitória, Vila Velha, Viana, Cariacica, Serra, São Mateus, Guarapari e Cachoeiro de Itapemirim) 72,2\% (372/515).

A forma pulmonar é a predominante em todo o estado, correspondendo a $68,4 \%(352 / 515)$ dos casos. Em relação à cultura de escarro e outro material, 76,9\% (396/515) e 65,8\% (339/515), respectivamente, não foram realizados e, aproximadamente, $9 \%(47 / 515)$ estão em andamento. O maior $(8,2 \%)$ índice de positividade foi em relação à cultura de outro material. $\mathrm{O}$ raio $\mathrm{X}$ foi suspeito em $80,9 \%$ dos casos e o teste tuberculínico foi reativo em $45,2 \%$ (233/515). Na variável tratamento, mais de $90 \%$ dos casos foram classificados como caso novo. Em relação à situação de encerramento, a maior taxa de cura foi encontrada nos municípios não prioritários; no entanto, todos eles tiveram taxa de cura superior a $60 \%$. O índice de tuberculose multirresistente foi de 4 (0,8\%) casos nos municípios de Serra e Vitória.

O município de Vitória apresenta um perfil diferente dos demais, pois mostra um menor percentual de cura, maior percentual de óbito e mudança diagnóstica. Vale ressaltar que Vitória é a capital do Estado e conta com o Hospital Infantil Nossa Senhora da Glória como referência para o diagnóstico de tuberculose infantil, onde se internam os casos mais graves.

No Estado do Espírito Santo, dos 77 municípios estudados, 27 não notificaram nenhum caso e 18 municípios apresentaram um caso de tuberculose infantil (Figura 1A).

Os municípios de Domingos Martins e Vitória apresentaram, respectivamente, a menor $(10,8)$ e a maior $(94,36)$ taxa de incidência bruta (por 100 mil habitantes), no período do estudo.

O cálculo da média móvel (Figura 1B) apresenta taxas de incidência ajustadas para os municípios. Dos 27 municípios com incidência igual a zero, somente quatro permaneceram com 
TABELA 1 - Caracterização dos sujeitos da pesquisa. Espírito Santo, 2000-2007 (no=515).

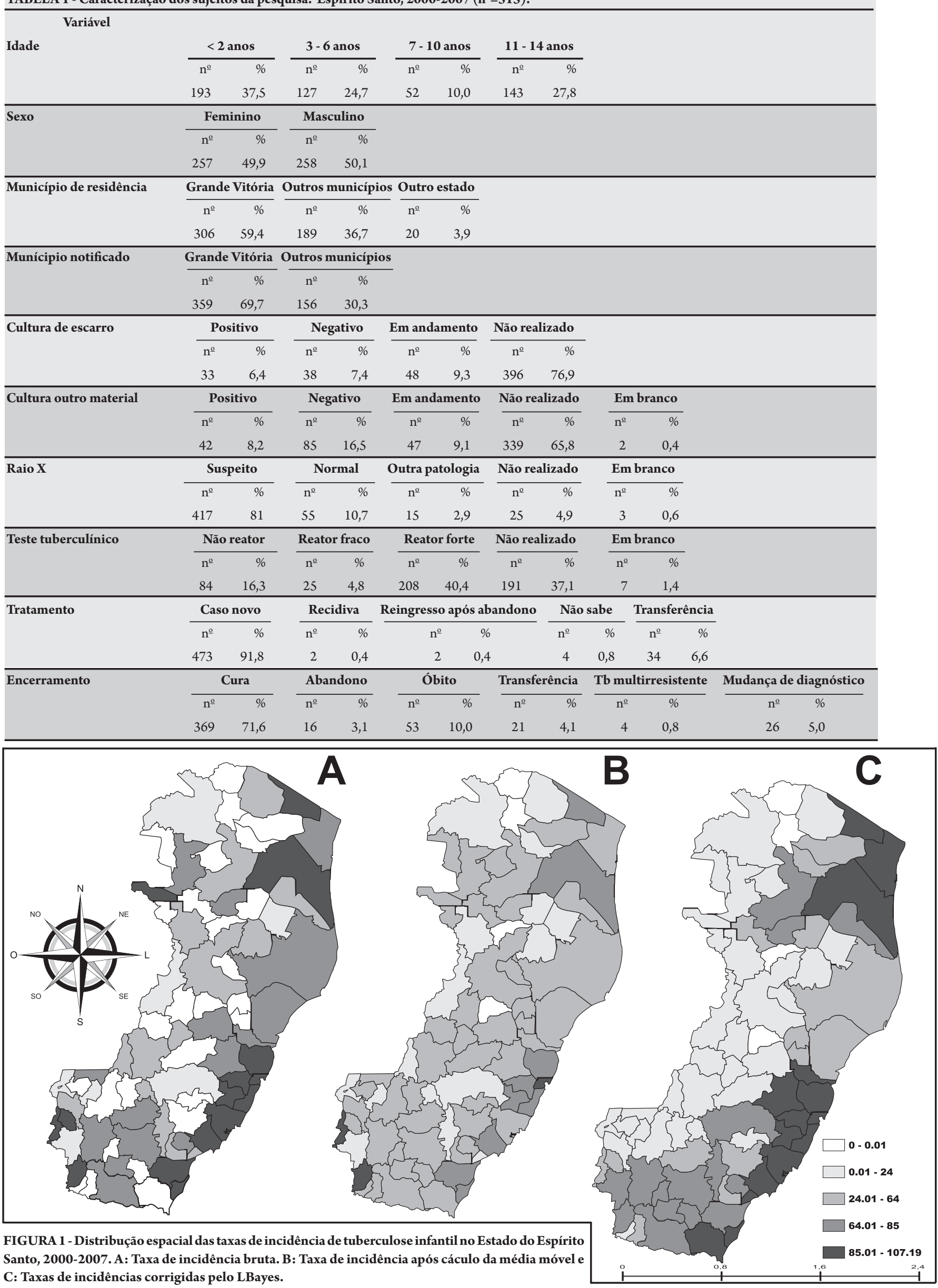


o número ajustado igual a zero: Mucurici e Ponto Belo, localizados ao sul do Estado, e São Domingos do Norte e São Roque do Canaã, localizados na região central do estado.

A Figura 1C exibe as taxas corrigidas pelo método LEBayes. Observa-se suavização das taxas do mapa, onde regiões de alta e baixa incidência da tuberculose infantil são evidenciadas. Destacamse regiões com altas taxas de incidência (a região metropolitana de Vitória e a região nordeste) e as de baixa taxa de incidência (região serrana) circuladas no mapa.

Por meio do cálculo do LISA (local spatial analysis), comprovouse a existência de padrões espaciais de distribuição locais. Os valores maiores que $95 \%$ são identificados por apresentarem dependência em relação à taxa observada nas áreas limítrofes. Na Figura 2, identificam-se áreas de alta incidência encontradas na região metropolitana da Grande Vitória, região sudoeste e no Município de Conceição da Barra. Na região noroeste, e nos Municípios de Colatina e Rio Bananal, baixas taxas de incidência encontram-se associadas.

O Índice de Moran, a partir das taxas estimadas pela média móvel (com os quatro municípios com zero casos), apresentou valor de $0,238214(p<0,001)$ o que mostra existir dependência entre as taxas de incidência dos municípios com padrões semelhantes como identificados no método LISA.

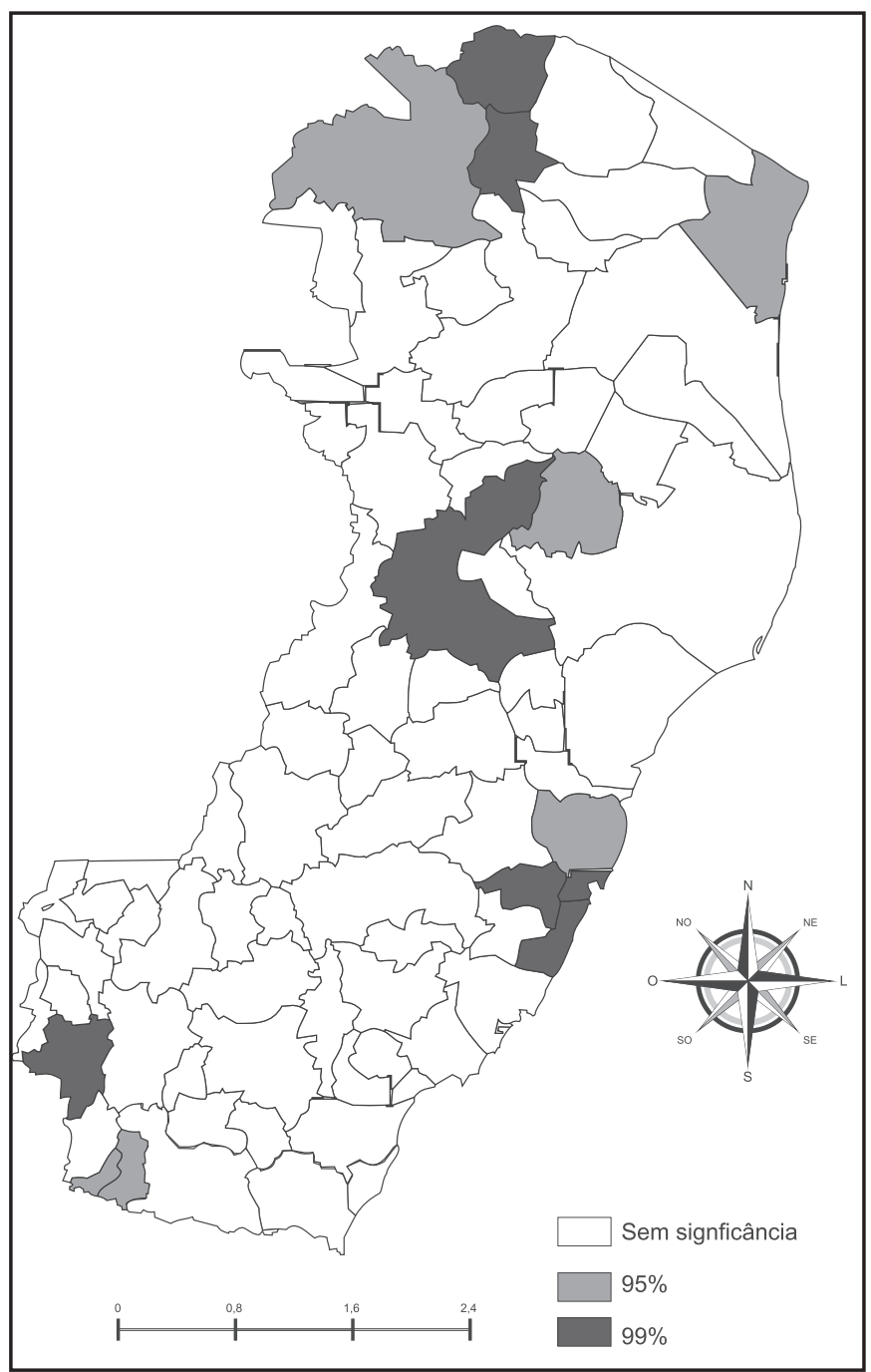

FIGURA 2 - Distribuição Espacial das regiões com significativa dependência espacial. 2000-2007.

\section{DISCUSSÃo}

O perfil epidemiológico de crianças e adolescentes foi realizado a fim de se conhecer a população do estudo.

Em relação à idade, tivemos maior porcentagem, na faixa etária entre 1 e 5 anos, seguida de uma queda entre 10 e 14 anos; e a menor porcentagem observou-se na faixa etária entre 6 e 9 anos. Quanto à situação de encerramento, $71,6 \%$ tiveram como resposta cura, índice que só pode ser considerado satisfatório, segundo o Programa Nacional de Controle da Tuberculose, quando o mesmo atinge a porcentagem de $80 \%$. Tal índice pode ser explicado, possivelmente, em função da maior infraestrutura hospitalar, como o Hospital Infantil Nossa Senhora da Glória (HINSG) e o Hospital Universitário Cassiano Antônio de Moraes (HUCAM), para onde vão os casos mais graves ${ }^{14}$.

Em relação à análise espacial, a escolha do município como unidade de análise não é algo comum em análises espaciais dessa doença. Usualmente, os estudos são realizados em um município tendo como unidade de análise setores censitários, bairros ou região administrativas ${ }^{15,16}$. Em relação a tuberculose infantil, não foi encontrado nenhum trabalho específico com análise espacial.

Neste trabalho, foi possível identificar, a princípio, 27 (35\%) municípios com taxa de incidência bruta igual a zero. Em estudo sobre a endemia da tuberculose, em maiores de 15 anos, no Espírito Santo ${ }^{17}$, todos os municípios apresentaram incidência da doença maior que zero, o que poderia indicar a subnotificação do caso da tuberculose infantil, no município de origem, ou diagnóstico nos centros maiores, onde há maior acesso e maior capacidade diagnóstica.

Outro fator a se considerar é um conjunto de municípios com altas taxas de notificação da tuberculose infantil, em sua maioria, nos municípios prioritários para o controle da tuberculose. Isso reforça a existência de uma associação entre a endemia da tuberculose em adultos e a tuberculose infantil. Outros estudos com unidade de análise no indivíduo já demonstraram que a transmissão da tuberculose infantil se dá através dos contatos intradomiciliares sendo que, quanto maior a carga bacilar do caso, maior a chance da infecção pelo bacilo de Koch dos seus contatos ${ }^{18}$.

Os mapas de autocorrelação espacial apontam os municípios com maiores taxas de incidência de tuberculose infantil indicando espacialmente locais onde podem estar ocorrendo infecção recente da doença ${ }^{19}$. A interrupção da transmissão recente e o controle da tuberculose infantil, pontos convergentes para o controle da tuberculose em países em desenvolvimento, ainda se apresentam como os grandes desafios da saúde pública.

Este estudo demonstra que a metodologia de análise espacial foi adequada, para o ajuste de áreas pequenas com baixa incidência de doença, além de identificar possíveis áreas de transmissão recente da doença. Por fim, é importante ressaltar que o conhecimento de locais prioritários para o controle, como demonstrado por este trabalho, pode auxiliar a gestão pública na diminuição das iniquidades em saúde e permitir uma otimização dos recursos e das equipes no controle da tuberculose infantil.

\section{CONFLITO DE INTERESSE}

Os autores declaram não haver nenhum tipo de conflito de interesse no desenvolvimento do estudo. 


\section{SUPORTE FINANCEIRO}

Este estudo recebeu apoio financeiro através do Edital MCT/ CNPq/MS-SCTIE-DECIT 25/2006- Estudo de Doenças Negligenciadas e do International Clinical, Operational and Health Services Research and Training Award, Edital ICOHRTA 5 U2R TW006883-02.

\section{REFERÊNCIAS}

1. World Health Organization. WHO Report 2008. Global Tuberculosis Control. Surveillance, Planning, Financing. WHO/HTM/TB: Genebra; 2008.

2. Starke JR, Jacobs RF, Jereb J. Resurgences of tuberculosis in children. J Pediatr 1992; 120:839-855.

3. Sant'anna C, Mourgues LV, Ferrero F, Balanzat AM. Diagnóstico e terapêutica da tuberculose infantil - uma visão atualizada de um antigo problema. J Pediatr 2002; 78:(supl 2):205-214

4. Maciel ELN, Martinato AE, Bandeira CFR, Tonini MS, Dietze R, Ramos MC $O$ perfil epidemiológico da Tuberculose em crianças e adolescentes em menores de 15 anos na Grande Vitória-ES, Brasil, no período de 1990-2001. Cad Saude Colet 2006; 14:81-94.

5. Houwert KA, Borggreven PA, Schaaf HS, Nel E, Donald PR, Stolk J. Prospective evaluation of World Heath Organization criteria to assist diagnosis of tuberculosis in children. Eur Respir J 1998; 11:1116-1120.

6. Nair PM, Philip E. A Scoring system for the diagnosis of tuberculosis in Children. Indian Pediatr 1981; 18:299-303.

7. Migliori GB, Borghesi A, Rossanigo P, Adriko C, Neri M, Santini S, et al. Proposal of an improved scores method for the diagnosis of pulmonary tuberculosis in childhood in developing countries. Int J Tuberc Lung Dis 1992; 73:145-149.

8. Fourie PB, Becker PJ, Festenstein F, Migliori GB, Alcaide J, Antunes M, et al. Procedures for developing a simple scoring method based on unsophisticated criteria for screening children for tuberculosis. Int J Tuberc Lung Dis 1998; 2:116-123.

9. Ministério da Saúde. Controle de Tuberculose: uma proposta de integração ensino - serviço. $5^{a}$ ed. Fundação Nacional de Saúde. Centro de Referência Prof. Hélio Fraga. Rio de Janeiro. Sociedade Brasileira de Pneumologia e Tisiologia; 2002

10. GEOBASES Espírito Santo. [Internet]. Disponível em: http://www.ijsn.es.gov. br/follow.asp?urlframe=geobases/index.htmIJSN. [Acessado em 01 de julho de 2008].

11. Ministério da Saúde. Sistema Nacional de Vigilância em Saúde: relatório de situação: Espírito Santo. Secretaria de Vigilância em Saúde. Brasília: Ministério da Saúde; 2005.

12. Bailey TC, Gatrell AC. Interactive Spatial Data Analysis. Essex: Longman; 1995

13. Anselin L. Local indicators of spatial Asssociation - LISA. Geogr Anal 1995; 27:93-115.

14. Costa MCN, Teixeira MGLC. A concepção do "espaço" na investigação metodológica. Cad Saude Publica 1999; 5:271-279.

15. Mota FF, Vieira-da-Silva LM, Paim JS, Costa MCN. Distribuição espacial da mortalidade por tuberculose em Salvador, Bahia, Brasil. Cad Saude Publica 2003; 19:915-922.

16. Vieira RC. A endemia de Tuberculose e seus determinantes socioeconômicos no Espírito Santo: Uma análise de dados espaciais. [Dissertação]. [Vitória, ES]: Universidade Federal do Espírito Santo; 2006.

17. Maciel ELN, Dietze R, Silva RECF, HadadDJ, Struchiner CJ. Avaliação do sistema de pontuação para o diagnóstico da tuberculose na infância preconizado pelo Ministério da Saúde, Brasil. Cad Saude Publica 2008; 24:402-408.

18. Pineda NS, Pereira SM, Matos ED, Barreto ML. Quimioprofilaxia na prevenção da tuberculose. J Bras Pneumol 2004; 30:485-495.

19. Newton SM, Brent AJ, Anderson S, Whittaker E, Kampmann B. Paediatrics tuberculosis. Lancet Infect Dis 2008; 8:498-510. 